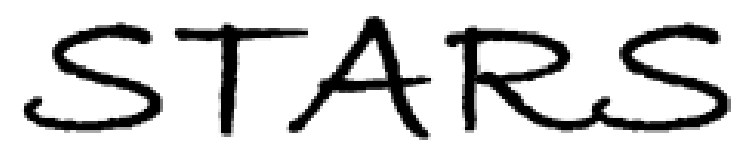

University of Central Florida

STARS

$1-1-2004$

\title{
Time-reversal imaging with multiple signal classification considering multiple scattering, between the targets
}

Fred K. Gruber

University of Central Florida

Edwim A. Marengo

Anthony J. Devaney

Find similar works at: https://stars.library.ucf.edu/facultybib2000

University of Central Florida Libraries http://library.ucf.edu

This Article is brought to you for free and open access by the Faculty Bibliography at STARS. It has been accepted for inclusion in Faculty Bibliography 2000 s by an authorized administrator of STARS. For more information, please contactSTARS@ucf.edu.

\section{Recommended Citation}

Gruber, Fred K.; Marengo, Edwim A.; and Devaney, Anthony J., "Time-reversal imaging with multiple signal classification considering multiple scattering, between the targets" (2004). Faculty Bibliography 2000 s. 4388.

https://stars.library.ucf.edu/facultybib2000/4388

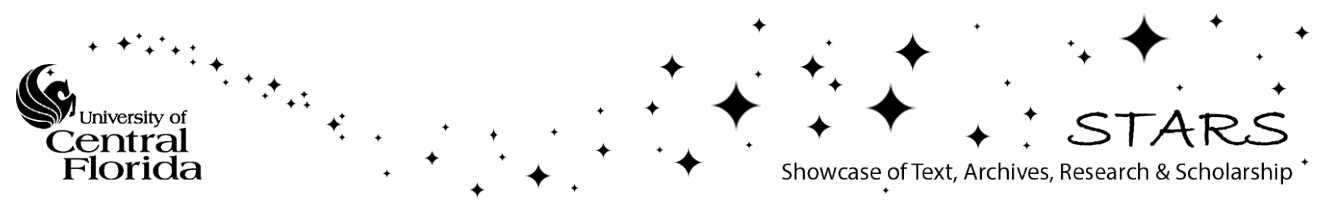




\section{Time-reversal imaging with multiple signal classification considering multiple scattering between the targets}

Fred K. Gruber, Edwin A. Marengo, and Anthony J. Devaney

Citation: The Journal of the Acoustical Society of America 115, 3042 (2004); doi: 10.1121/1.1738451

View online: https://doi.org/10.1121/1.1738451

View Table of Contents: https://asa.scitation.org/toc/jas/115/6

Published by the Acoustical Society of America

\section{ARTICLES YOU MAY BE INTERESTED IN}

Time-reversal-based imaging and inverse scattering of multiply scattering point targets

The Journal of the Acoustical Society of America 118, 3129 (2005); https://doi.org/10.1121/1.2042987

Transmission mode time-reversal super-resolution imaging

The Journal of the Acoustical Society of America 113, 2742 (2003); https://doi.org/10.1121/1.1566975

Experimental subwavelength localization of scatterers by decomposition of the time reversal operator interpreted as a covariance matrix

The Journal of the Acoustical Society of America 114, 235 (2003); https://doi.org/10.1121/1.1568759

Decomposition of the time reversal operator: Detection and selective focusing on two scatterers

The Journal of the Acoustical Society of America 99, 2067 (1996); https://doi.org/10.1121/1.415393

Time-reversal multiple signal classification in case of noise: A phase-coherent approach

The Journal of the Acoustical Society of America 130, 2024 (2011); https://doi.org/10.1121/1.3626526

The iterative time reversal process: Analysis of the convergence

The Journal of the Acoustical Society of America 97, 62 (1995); https://doi.org/10.1121/1.412285

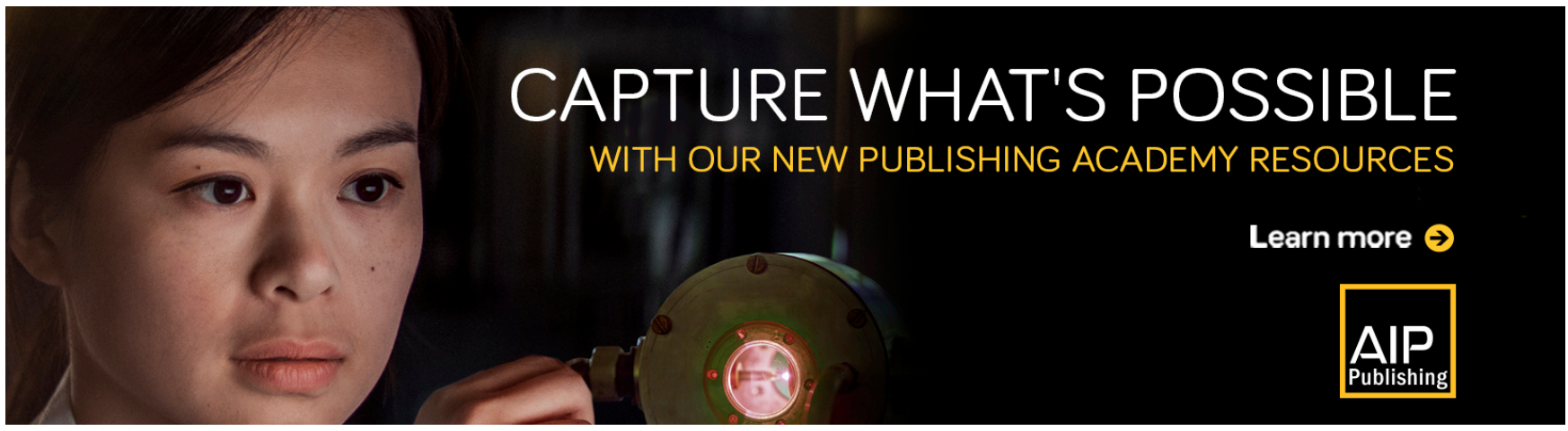




\title{
Time-reversal imaging with multiple signal classification considering multiple scattering between the targets
}

\author{
Fred K. Gruber ${ }^{\mathrm{a})}$ \\ Department of Industrial Engineering and Management Systems, University of Central Florida, Orlando, \\ Florida 32816 and Department of Electrical Engineering, Technological University of Panama, \\ Apartado 6-2894, Panama City, Panama
}

Edwin A. Marengo

Department of Electrical and Computer Engineering, Northeastern University, Boston, Massachusetts 02115 and Department of Electrical Engineering, Technological University of Panama, Apartado 6-2894, Panama City, Panama

Anthony J. Devaney

Department of Electrical and Computer Engineering, Northeastern University, Boston, Massachusetts 02115

(Received 29 October 2003; revised 23 February 2004; accepted 13 March 2004)

\begin{abstract}
The time-reversal imaging with multiple signal classification method for the location of point targets developed within the framework of the Born approximation in Lehman and Devaney ["Transmission mode time-reversal super-resolution imaging," J. Acoust. Soc. Am. 113, 2742-2753 (2003)] is generalized to incorporate multiple scattering between the targets. It is shown how the same method can be used in the location of point targets even if there is multiple scattering between them. On the other hand, both the conventional images and the calculated values of the target scattering amplitudes are scattering model-dependent. (C) 2004 Acoustical Society of America.
\end{abstract}

[DOI: $10.1121 / 1.1738451]$

PACS numbers: $43.60 . P t, 43.35 . Z c[\mathrm{JCB}]$

Pages: $3042-3047$

\section{INTRODUCTION}

Time-reversal (TR) methods have been of interest for a number of years, with applications ranging from medicine, to communications, to radar, and to imaging in general. ${ }^{1-6} \mathrm{~A}$ particular TR technique that has received much attention is a TR imaging with multiple signal classification (MUSIC) method for the location of point targets (whose size is much smaller than the wavelength) established first for coincident arrays, ${ }^{7}$ and elaborated further in a recent generalization to noncoincident arrays and nonreciprocal media ${ }^{8}$ and a recent super-resolution analysis based on real data. ${ }^{9}$ This TR imaging with MUSIC method blends the original TR focusing ${ }^{10,11}$ with signal subspace methods, in particular, with the MUSIC estimation technique. In its basic form the method is designed to deduce the location of point targets embedded in a known background medium from knowledge of the measured multistatic response matrix $K$ of an acoustic or electromagnetic transceiver array actively interrogating the targets. It provides a powerful approach for locating targets in both homogeneous and heterogeneous backgrounds, especially in cases of closely spaced targets and/or very sparse transceiver arrays. However, these developments are based on the (distorted wave) Born approximation. The present letter addresses the corresponding generalization if there is nonnegligible multiple scattering between the targets.

a)Electronic mail: fgruber@mail.ucf.edu

\section{GENERALIZATION INCLUDING MULTIPLE SCATTERING BETWEEN THE TARGETS}

We consider an array of $N$ time-harmonic transceivers located at the space points $\mathbf{R}_{j}$, for $j=1,2, \ldots, N$. Each transceiver can radiate into a reciprocal propagating medium in which are embedded $M<N$ point targets having unknown positions $\mathbf{X}_{m}$ and unknown target scattering amplitudes $\tau_{m}$, for $m=1,2, \ldots, M$. For simplicity, we model the field radiated by the $j$ th transceiver as the scalar field $G\left(\mathbf{r}, \mathbf{R}_{j}\right) e_{j}$ where $G\left(\mathbf{r}, \mathbf{r}^{\prime}\right)$ is the Green function of the propagating medium (in principle, the "medium" can include the transceivers themselves so inter-transceiver coupling can be built in this Green function) and $e_{j}$ is the excitation applied to the transceiver.

\section{A. Review of the TR imaging with MUSIC method}

Under the Born approximation the TR imaging with MUSIC method is based on the fact that, for fewer targets than transceivers, the space $\mathcal{C}^{N}$ of voltage vectors applied to the $N$-transceiver array can be decomposed into the direct sum $\mathcal{C}^{N}=\mathcal{S} \oplus \mathcal{N}$, where the signal subspace $\mathcal{S}$ is orthogonal to the noise subspace $\mathcal{N}$, where $\mathcal{S}$ is spanned by the principal eigenvectors $\mu_{i}$ of the TR matrix $T=K^{\dagger} K$ (where $\dagger$ denotes the adjoint) having nonzero eigenvalues (if there are $M<N$ targets then there are at most $M$ such eigenvalues indexed from 1 to $M$ ) whereas $\mathcal{N}$ is spanned by the eigenvectors $\mu_{i}$ of $T$ having zero eigenvalues (if there are $M<N$ targets then there are at least $N-M$ such eigenvalues indexed from $M$ +1 to $N)$. The key result is that the TR matrix $T$ is essentially a projection operator onto the space spanned by the complex conjugates $g_{m}^{*}$ of the Green function vectors defined 
as $g_{m}=\left[G\left(\mathbf{R}_{1}, \mathbf{X}_{m}\right), G\left(\mathbf{R}_{2}, \mathbf{X}_{m}\right), \ldots, G\left(\mathbf{R}_{N}, \mathbf{X}_{m}\right)\right]^{t}$ where $t$ denotes the transpose, so that in general the principal eigenvectors of $T$ are linear combinations of the $g_{m}^{*}$ 's. It follows from this and the orthogonality of the signal and noise subspaces that the target locations must correspond to the poles in the MUSIC pseudospectrum

$$
D\left(\mathbf{X}_{p}\right)=\frac{1}{\sum_{i=M+1}^{N}\left|\left\langle\mu_{i}^{*} \mid g_{p}\right\rangle\right|^{2}},
$$

where for all $i=M+1, M+2, \ldots, N$ the inner product $\left\langle\mu_{i}^{*} \mid g_{p}\right\rangle \equiv \mu_{i}^{t} g_{p}=0$ whenever $\mathbf{X}_{p}$ is the actual location of one of the targets.

\section{B. Our main results}

Essentially, what we show next is that not only for the Born-approximated model but also for the more general case when there is multiple scattering between the targets the TR matrix $T$ is a projection operator onto the space spanned by the complex conjugates $g_{m}^{*}$ of the Green function vectors $g_{m}$ so that in general the principal eigenvectors of $T$ are linear combinations of the $g_{m}^{*}$, s. It follows that the same TR imaging with MUSIC method described in connection with Eq. (1) can be used in the location of point targets even if there is multiple scattering between them. On the other hand, both the conventional TR images, ${ }^{10,11}$ corresponding to backpropagations of the principal eigenvectors, and the calculated values of the target scattering amplitudes are scattering model-dependent.

\section{Formulation}

The exact scattered field $\psi_{j}^{(s)}(\mathbf{r})$ at position $\mathbf{r}$ due to the excitation of the $j$ th transceiver alone can be expressed as the Newman series

$$
\begin{aligned}
\psi_{j}^{(s)}(\mathbf{r})= & \sum_{m=1}^{M} G\left(\mathbf{r}, \mathbf{X}_{m}\right) \tau_{m} G\left(\mathbf{X}_{m}, \mathbf{R}_{j}\right) e_{j} \\
& +\sum_{m=1}^{M} \sum_{m^{\prime}=1}^{M} G\left(\mathbf{r}, \mathbf{X}_{m}\right) \tau_{m} G\left(\mathbf{X}_{m}, \mathbf{X}_{m^{\prime}}\right) \\
& \times \tau_{m^{\prime}} G\left(\mathbf{X}_{m^{\prime}}, \mathbf{R}_{j}\right)\left(1-\delta_{m, m^{\prime}}\right) e_{j} \\
& +\sum_{m=1}^{M} \sum_{m^{\prime}=1}^{M} \sum_{m^{\prime \prime}=1}^{M} G\left(\mathbf{r}, \mathbf{X}_{m}\right) \tau_{m} G\left(\mathbf{X}_{m}, \mathbf{X}_{m^{\prime}}\right) \\
& \times \tau_{m^{\prime}} G\left(\mathbf{X}_{m^{\prime}}, \mathbf{X}_{m^{\prime \prime}}\right) \tau_{m^{\prime \prime}} G\left(\mathbf{X}_{m^{\prime \prime}}, \mathbf{R}_{j}\right) \\
& \times\left(1-\delta_{m, m^{\prime}}\right)\left(1-\delta_{m^{\prime}, m^{\prime \prime}}\right) e_{j}+\cdots
\end{aligned}
$$

where $\delta_{\text {., }}$ denotes the Kronecker delta. The first sum in Eq. (2) corresponds to the Born-approximated case, the second sum corresponds to the second-order scattering contribution, and so on.

The multistatic response matrix $K$, whose entry $K_{l, j}$ is defined as the value of the scattered field detected at the $l$ th transceiver (in receive mode) due to the unit excitation at the $j$ th transceiver (in transmit mode), can be shown from Eq. (2) to be given by (see also Borcea et $_{\text {al. }}{ }^{12}$ )

$$
K=\sum_{m=1}^{M} g_{m} \tau_{m} \Lambda_{m}^{t}
$$

where

$$
\begin{aligned}
\Lambda_{m}^{t}= & g_{m}^{t}+\sum_{m^{\prime}=1}^{M} G\left(\mathbf{X}_{m}, \mathbf{X}_{m^{\prime}}\right) \tau_{m^{\prime}}\left(1-\delta_{m, m^{\prime}}\right) g_{m^{\prime}}^{t} \\
& +\sum_{m^{\prime}=1}^{M} \sum_{m^{\prime \prime}=1}^{M} G\left(\mathbf{X}_{m}, \mathbf{X}_{m^{\prime}}\right) \tau_{m^{\prime}} G\left(\mathbf{X}_{m^{\prime}}, \mathbf{X}_{m^{\prime \prime}}\right) \tau_{m^{\prime \prime}}(1 \\
& \left.-\delta_{m, m^{\prime}}\right)\left(1-\delta_{m^{\prime}, m^{\prime \prime}}\right) g_{m^{\prime \prime}}^{t}+\cdots
\end{aligned}
$$

(the approximation $\Lambda_{m}^{t} \simeq g_{m}^{t}$ yields the multistatic response matrix in the Born-approximated model). It follows from Eq. (3) that the TR matrix $T=K^{\dagger} K=K^{*} K$, where the second equality follows from reciprocity, can be expressed as

$$
T=\sum_{m=1}^{M} \sum_{m_{0}=1}^{M} g_{m}^{*} \tau_{m}^{*}\left\langle\Lambda_{m} \mid g_{m_{0}}\right\rangle \tau_{m_{0}} \Lambda_{m_{0}}^{t} .
$$

Put in this form, the TR matrix $T$ is identified to be under the most general multiple scattering conditions a projection operator onto the space spanned by the complex conjugates $g_{m}^{*}$ of the Green function vectors $g_{m}$ so that in general the principal eigenvectors of $T$ are linear combinations of the $g_{m}^{*}$, $s$, with the music pseudospectrum $D\left(\mathbf{X}_{p}\right)$ in Eq. (1) having poles at the locations of the targets, as desired. We illustrate this generalization next for a few canonical examples based on the free space Green function in two-dimensional space.

\section{TR imaging with MUSIC in the second-order scattering case}

In the following computer illustrations we consider the second-order scattering approximation of the preceding general theory. Our main goal is to show that despite the presence of non-negligible second-order scattering the TR imaging with MUSIC method works well in predicting the target locations. We will introduce a normalized quantity, to be referred to as "the second-order scattering contribution factor," and defined as $\eta=\mid \operatorname{Tr}\left[\left(K_{2 \text { nd }}-K_{\text {Born }}\right) *\left(K_{2 \text { nd }}\right.\right.$ $\left.\left.-K_{\text {Born }}\right)\right] / \operatorname{Tr}\left(T_{\text {Born }}\right)$, where $\operatorname{Tr}$ denotes the trace, $K_{\text {Born }}$ and $T_{\text {Born }}$ are the multistatic response matrix and the TR matrix, respectively, assuming the Born approximation, and $K_{2 \mathrm{nd}}$ is the multistatic response matrix for a more general secondorder scattering model, as a way to quantify the global effect of the second-order scattering contribution relative to that of the first-order scattering term alone as seen by the array in receive mode. This factor measures the deviation of the data matrix $(K)$, as determined by the energy norm or inner product metric, relative to the purely Born-approximated vector, due to the second-order scattering contribution.

Figures 1 and 2 show plots in gray color map of the MUSIC pseudospectra under a second-order scattering model for a number of transceiver and target configurations. Next to each MUSIC pseudospectrum plot we have provided another plot showing the corresponding simulation geometry where the transceiver locations are indicated with triangles while the target locations are indicated with asterisks. All dimensions are relative to the wavelength $\lambda$ (which equals 

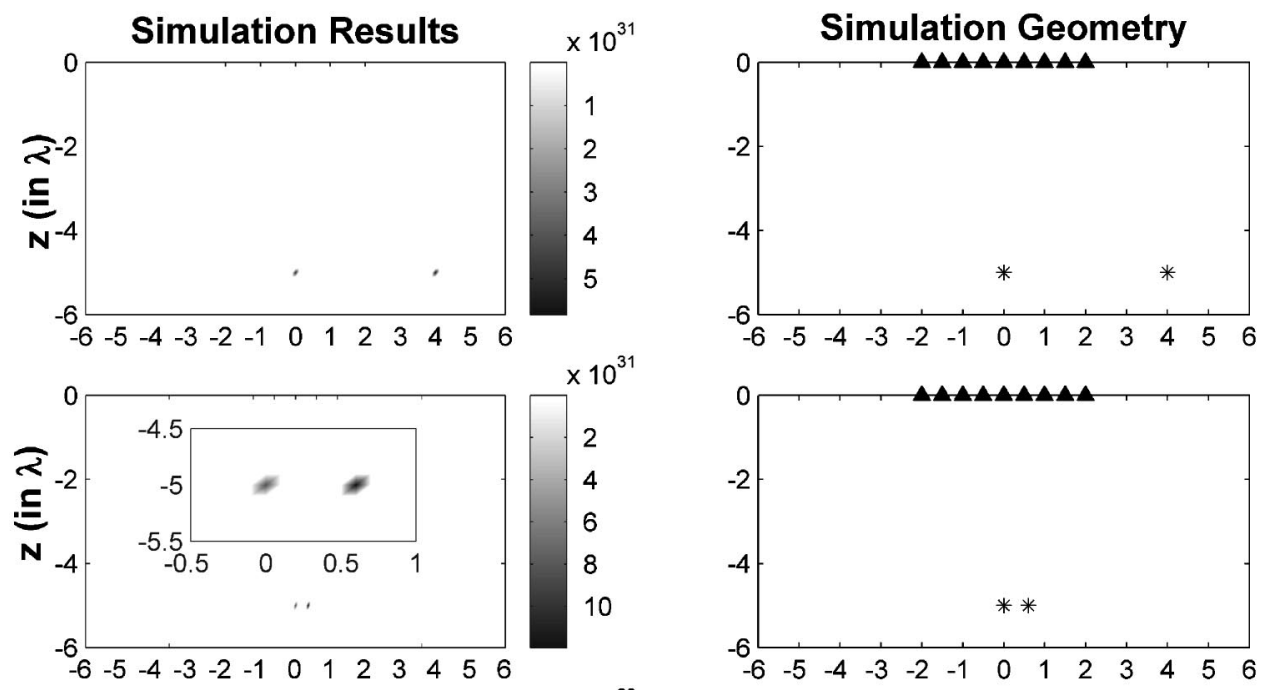

FIG. 1. Reconstructed image with an inverse gray color map for the secondorder scattering simulation pseudospectrum for a two target configuration. Three target separations are shown: $4 \lambda$ (top), $0.6 \lambda$ (middle), and $0.3 \lambda$ (bottom). A linear scale was used for the horizontal and vertical axes.
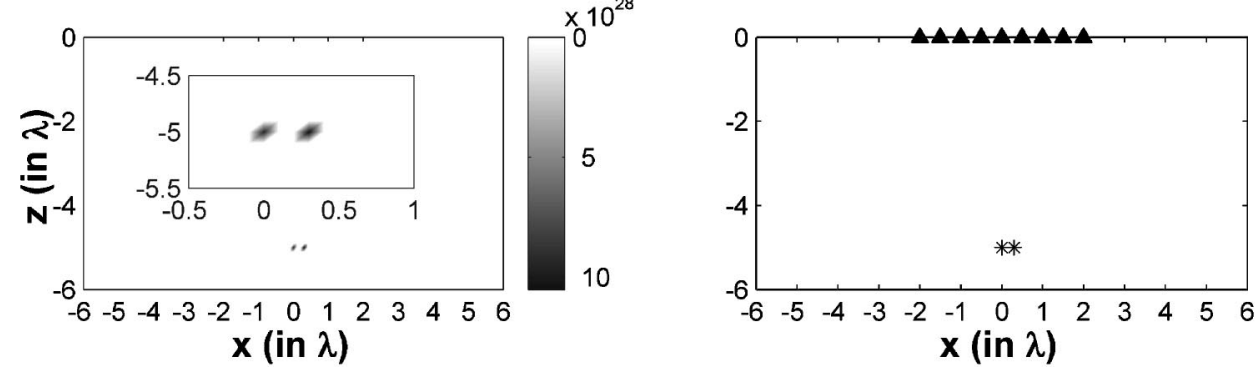

1), and all targets have unit scattering amplitude. The plot in Fig. 1 illustrates the super-resolution capabilities of the technique ${ }^{9}$ since even for a target separation as small as $0.3 \lambda$ the target locations remain sharply resolved [the method was found to work for yet smaller target separations, in particular,
$0.1 \lambda$ (results not shown)]. For the $0.6 \lambda$ and $4 \lambda$ target separation cases the second-order contribution factor is not very strong ( $\eta=0.17$ and $\eta=0.03$, respectively). However, for the $0.3 \lambda$ and $0.1 \lambda$ target separation cases, the second-order contribution factor is $\eta=0.33$ and $\eta=0.89$, respectively.
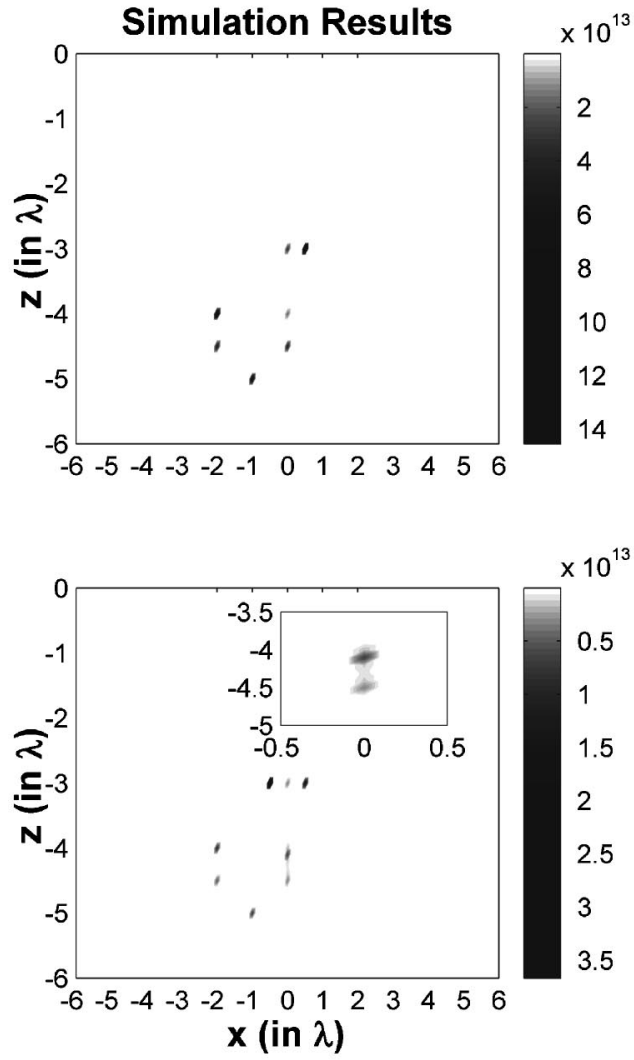

Simulation Geometry
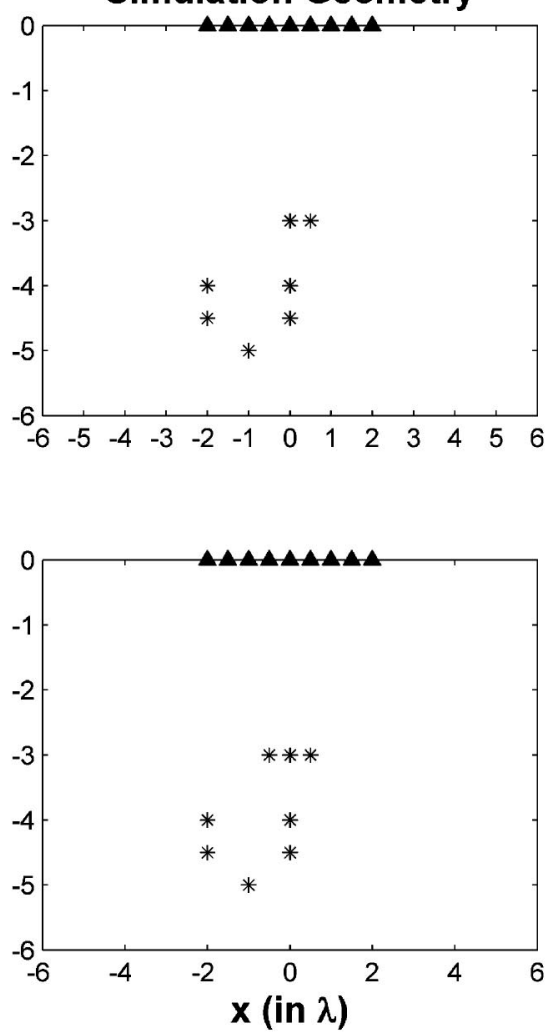

FIG. 2. Reconstructed image with an inverse gray color map for the secondorder scattering simulation pseudospectrum for 7 and 8 targets in a $J$ conformation. A linear scale was used for the horizontal and vertical axes. 

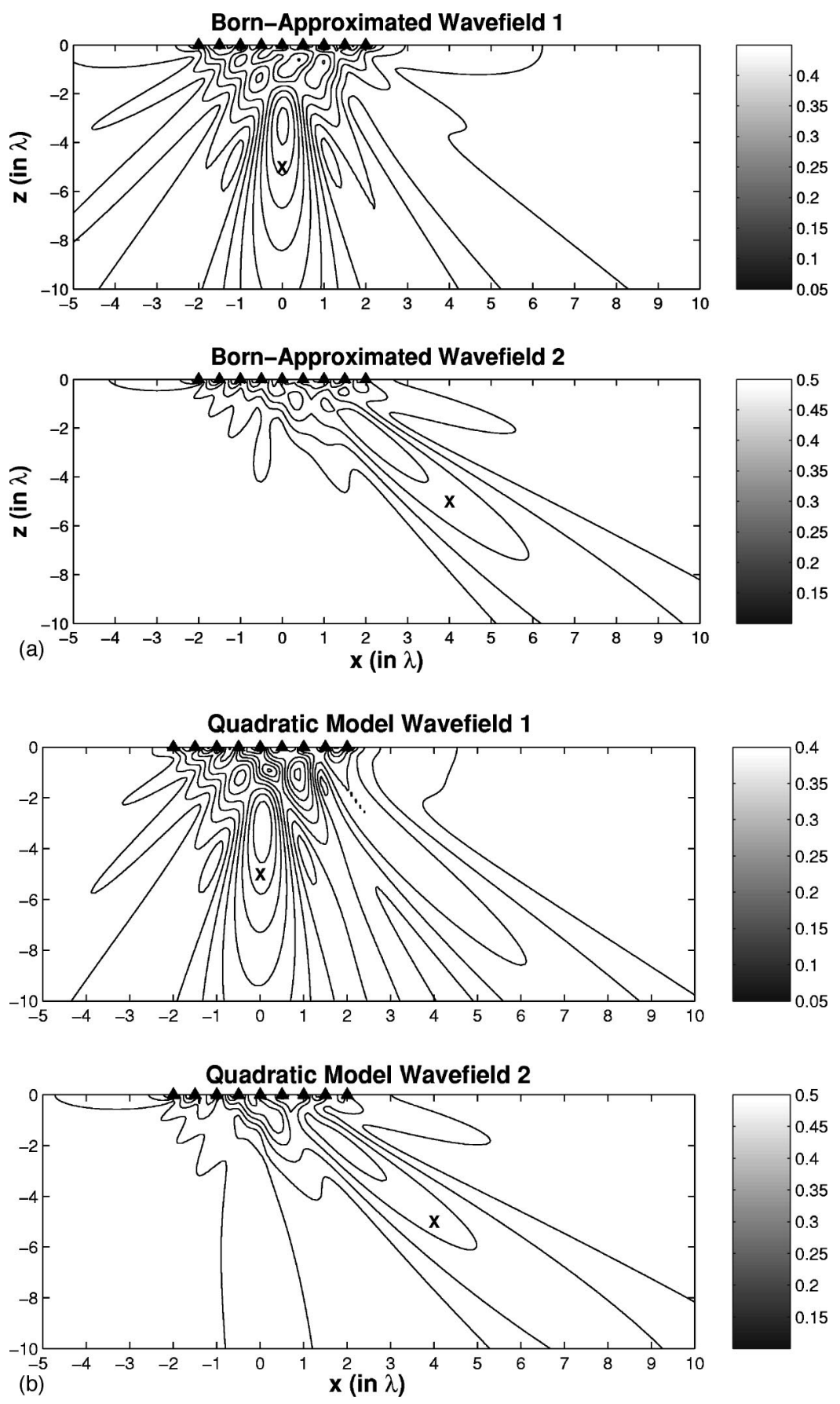

This means that in the $0.3 \lambda$ target separation configuration the amount of additional signal due to the presence of second-order scattering is of the order of the Bornapproximated signal alone as perceived by the array in receive mode. In the $0.1 \lambda$ target separation case the presence of second-order scattering is even more dominant. Yet the TR imaging with MUSIC was found to succeed in determining the target locations. More complicated seven and eight target configurations forming a $J$ and having significant secondorder scattering contribution factors of $\eta=0.31$ and $\eta$ $=0.30$, respectively, are considered in Fig. 2 . The method works well in predicting the target locations but for the eight target case the prediction for one of the target locations, in particular, the one at the plot coordinates $(0,-4 \lambda)$, has a slight error which we attribute to computational sensitivity
FIG. 3. (a) Conventional, backpropagated images of the two principal eigenvectors corresponding to the $4 \lambda$ target separation geometry in Fig. 1. Results for the Bornapproximated model. A linear scale was used for the horizontal and vertical axes. (b) Corresponding results for the second order scattering model. under reduced noise subspace conditions (in this extreme case when $M=8$ and $N=9$ the noise subspace has a single eigenvector).

\section{E. Time-reversed images: Born-approximated versus second-order scattering cases}

The field radiated by the array due to excitation with an arbitrary voltage vector is $\psi(\mathbf{r})=\sum_{j=1}^{N} G\left(\mathbf{r}, \mathbf{R}_{j}\right) e_{j}$. In the original TR focusing one obtains backpropagated images $\psi(\mathbf{r})$ of the targets by replacing the arbitrary input voltage vectors in this expression with the principal eigenvectors of the TR matrix. If there is no second-order scattering between the targets then the principal eigenvectors are precisely the complex conjugates of the Green function vectors and each 


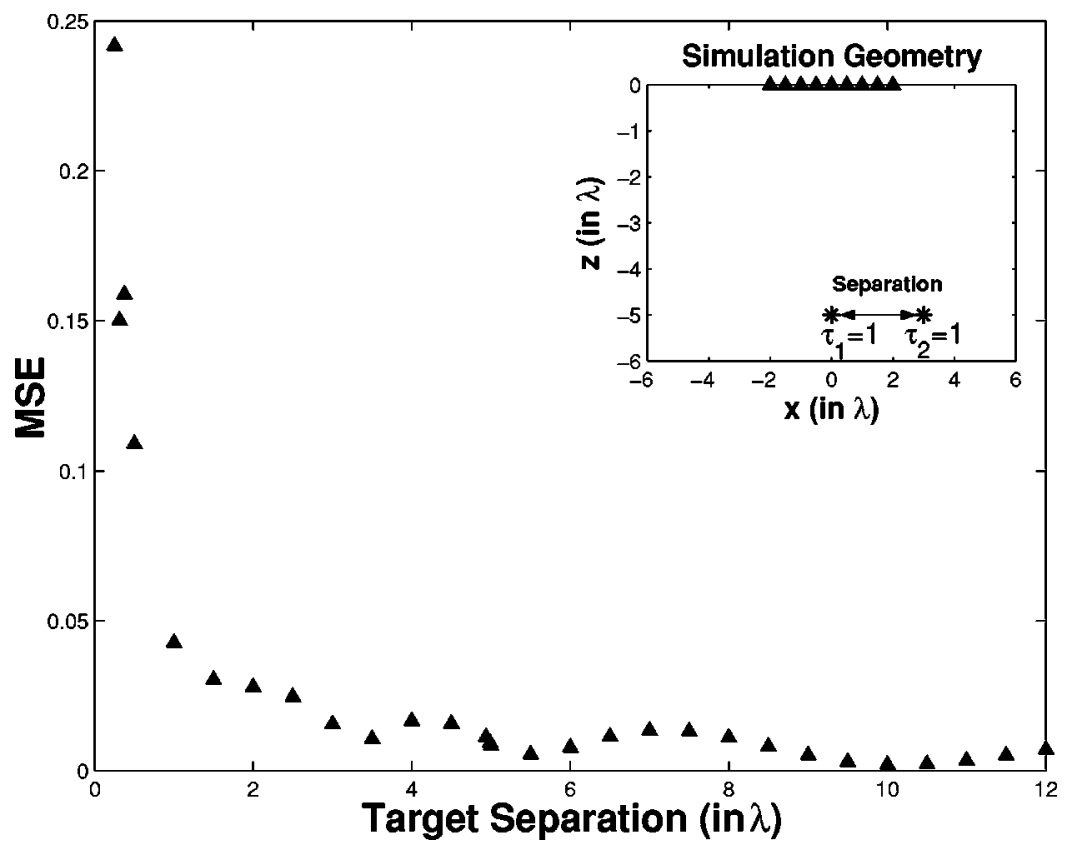

FIG. 4. MSE in scattering amplitude estimation, incurred if one uses the Born-approximated model instead of the more exact second-order scattering model, as a function of target separation for the general two target configuration used in Fig. 1.

one of them is associated with (focuses on) a particular target. Consequently, as shown in Fig. 3(a), corresponding to the $4 \lambda$ target separation case in Fig. 1, in the Bornapproximated case each "eigenimage" focuses on a particular, corresponding target (in the plots we have superimposed an $X$ in the location of the corresponding target). On the other hand, if there is second-order scattering between the targets, then the principal eigenvectors become linear combinations of the complex conjugates of the Green function vectors so that each eigenimage focuses in general on more than one target (this is somehow similar to the results in Ref. 11 for non-well-resolved targets). Figure 3(b) illustrates this effect for the second-order scattering case where each eigenimage is seen to focus on both targets.

\section{F. Target scattering amplitudes: Born-approximated versus second-order scattering cases}

In the general case including multiple scattering considered here, once the target locations are determined from the poles in the MUSIC pseudospectrum one can compute the unknown target scattering amplitudes by substituting the values of these locations in Eq. (3) and solving the resulting system of equations. In general, such computed values will depend on the scattering model employed, being more exact for a more exact scattering model. To illustrate this let us assume that in reality the response obeys a second-order scattering model. If we try to determine the scattering amplitudes considering a Born-approximated model then we will have model-induced errors. Figure 4 shows, again for the geometry in Fig. 1, the mean square error (MSE) in the estimation of the scattering amplitudes (as measured relative to the more exact second-order scattering model) as a function of target separation. The errors are significant (relative to the value $\tau_{1}=\tau_{2}=1$ ) for targets in close proximity, as expected (second-order scattering becomes then more prevalent).

\section{CONCLUSION}

In this letter we showed that the TR imaging with MUSIC method can be used to locate point targets not only in the usual framework of the Born approximation but also in more general nonlinear scattering frameworks where there is multiple scattering between the targets. This finding is important toward practical implementations of the TR technique facing multiple scattering effects. The super-resolution results of a previous paper ${ }^{9}$ can now be better characterized theoretically in the multiple scattering regime. They were also corroborated numerically for second-order scattering (see Fig. 1). The method was found to work well even for a large number of targets relative to the number of transceivers (see Fig. 2) although slight numerical challenges may arise as the number of targets approaches the number of transceivers (see the zooming part of Fig. 2). We showed how both the conventional, backpropagated images of the principal eigenvectors and the predicted values of the target scattering amplitudes are scattering model-dependent (see Figs. 3 and 4). In this the effect of scattering model is significant, particularly for targets in close proximity, as expected (see Fig. 4).

\section{ACKNOWLEDGMENT}

This work was supported in part by the United States Air Force Office of Scientific Research under Grant No. F4962002-1-0309.

${ }^{1}$ M. Fink, D. Cassereau, A. Derode, C. Prada, P. Roux, M. Tanter, J. L. Thomas, and F. Wu, "Time-reversed acoustics," Rep. Prog. Phys. 63, 1933-1995 (2000).

${ }^{2}$ G. Montaldo, P. Roux, A. Derode, C. Negreira, and M. Fink, "Generation of very high pressure pulses with 1-bit time reversal in a solid waveguide," J. Acoust. Soc. Am. 110, 2849-2857 (2001).

${ }^{3}$ J. S. Kim and K. C. Shin, "Multiple focusing with adaptive time-reversal mirror," J. Acoust. Soc. Am. 115, 600-606 (2004).

${ }^{4}$ A. Derode, A. Tourin, J. de Rosny, M. Tanter, S. Yon, and M. Fink, 
"Taking advantage of multiple scattering to communicate with timereversal antennas," Phys. Rev. Lett. 90, 014301 (2003).

${ }^{5}$ D. M. Fromm, C. F. Gaumond, J. F. Lingevitch, R. C. Gauss, and R. Menis, "Detection in shallow water using broadband-DORT," J. Acoust. Soc. Am. 114, 2399 (2003).

${ }^{6}$ L. Carin, H. Liu, T. Yoder, L. Couchman, B. Houston, and J. Bucaro, "Wideband time-reversal imaging of an elastic target in an acoustic waveguide," J. Acoust. Soc. Am. 115, 259-268 (2004).

${ }^{7}$ H. Lev-Ari and A. J. Devaney, "The time-reversal technique reinterpreted: Subspace-based signal processing for multi-static target location," IEEE Sensor Array and Multichannel Signal Processing Workshop, Cambridge, MA, 2000, pp. 509-513.
${ }^{8}$ S. K. Lehman and A. J. Devaney, "Transmission mode time-reversal super-resolution imaging," J. Acoust. Soc. Am. 113, 2742-2753 (2003).

${ }^{9}$ C. Prada and J. L. Thomas, "Experimental subwavelength localization of scatterers by decomposition of the time reversal operator interpreted as a covariance matrix," J. Acoust. Soc. Am. 114, 235-243 (2003).

${ }^{10}$ C. Prada, J. L. Thomas, and M. Fink, “The iterative time reversal process: Analysis of the convergence," J. Acoust. Soc. Am. 97, 62-71 (1995).

${ }^{11}$ C. Prada, S. Manneville, D. Spoliansky, and M. Fink, "Decomposition of the time reversal operator: Detection and selective focusing on two scatterers," J. Acoust. Soc. Am. 99, 2067-2076 (1996).

${ }^{12}$ L. Borcea, G. Papanicolaou, C. Tsogka, and J. Berryman, "Imaging and time reversal in random media," Inverse Probl. 18, 1247-1279 (2002). 